\title{
Mass Attenuation Coefficient Measurements of Some Nanocarbon Allotropes: A New Hope for Better Low Cost Less-Cumbersome Radiation Shielding Over A Wide Energy Range
}

\author{
E. RAJASEKHAR ${ }^{1}$, K.L. NARASIMHAM ${ }^{2}$, ADITYA D. KURDEKAR ${ }^{3}$ L.A. \\ AVINASH CHUNDURI ${ }^{4}$, SANDEEP PATNAIK ${ }^{4}$ AND K. VENKATARAMANIAH ${ }^{3 *}$ \\ ${ }^{1}$ Department of Physics, Rayalaseema University, Kurnool, A.P., India \\ ${ }^{2}$ Department of Physics, Kakinada Institute of Technology \& Science, Divilli, \\ Tirupathi (V) 533433, A.P.,India \\ ${ }^{3}$ Department of Physics, Sri Sathya Sai Institute of Higher Learning, Prashanthinilayam \\ 515134 A.P., India
}

${ }^{4}$ Andhra Pradesh Medtech Zone, AMTZ, Vishakhapatnam, 530045, A.P. India

Email: kvenkataramaniah@sssihl.edu.in

Received: September 12, 2017| Revised: November 09, 2017| Accepted: December 22, 2017

Published online: February 05, 2018

The Author(s) 2018. This article is published with open access at www.chitkara.edu.in/publications

\begin{abstract}
The mass attenuation coefficients of graphene, MWNTs and, SWNTs have been measured for gamma energy range 356 to $1332 \mathrm{keV}$ from the radioactive sources ${ }^{60} \mathrm{Co},{ }^{133} \mathrm{Ba}$ and ${ }^{137} \mathrm{Cs}$ using a well calibrated gamma ray spectrometer consisting of a $3^{\prime \prime} \mathrm{x} 3^{\prime \prime} \mathrm{NaI}(\mathrm{Tl})$ scintillation detector coupled to a PC based 8K nuclear Multi Channel Analyser (MCA). In an interesting way results showed that MWNTs had the highest values of mass attenuation coefficients indicating their potential use as the best shielding material.
\end{abstract}

Keywords: Graphene; SWCNTs; MWCNTs; Mass attenuation coefficient; $\mathrm{NaI}(\mathrm{Tl})$ detector.

\section{INTRODUCTION}

It is noteworthy that different types of Nano materials have been actively studied using X-ray absorption spectroscopy [1, 2].These works reveal the presence of an anomaly in X-ray absorption data, which arises from the polarization function due to the electron population fluctuation. In addition, the recent study on Berry-phase transition in deformed crystals by Sawada

\footnotetext{
Journal of Nuclear

Physics, Material

Sciences, Radiation and

Applications

Vol-5, No-2,

February 2018

pp. 311-317
} 
Rajsekhar, E Narasimham, KL

Kurdekar, AD

Chunduri, LAA

Patnaik, S

Venkataramaniah, $\mathrm{K}$ et al. indicates the necessity of carrying out further fundamental studies related to the interaction of X-rays with nano structured materials [3]. It is important to emphasize that CNTs possess a unique structure consisting of cylindrical tubules that could be nested (e.g., MWCNTs), and the interaction of radiation with these tubules appears to be very different when compared to interactions with planar or stacked graphene sheets. The microwave absorption of CNTs is usually discussed in terms of the electromagnetic wave theory, as referred in theoretical approaches[4-6]. Although an effective medium theory developed by Lagarkov and Sarychev could describe the microwave absorption properties of CNT composites but a clear explanation about the enhanced microwave absorption properties of CNTs has not been provided [4]. Graphene is a rapidly rising star on the horizon of materials science and condensed-matter physics. This strictly two-dimensional material exhibits exceptionally high crystal and electronic quality, despite its short history revealed an abundance of new physics and potential. More generally, graphene represents a conceptually new class of materials that are only one atom thick, and, on this basis, offers new inroads into low-dimensional physics that has never ceased to surprise and continues to provide a fertile ground for applications. It has been shown that Nanomaterials could convert microwave energy into light or heat in high yields. These studies demonstrate that Nanomaterials could exhibit outstanding electromagnetic effects [79]. In this study, an attempt is made to investigate the gamma ray shielding properties of graphene, multi-walled nano tubes (MWNTs) and singlewalled nanotubes (SWNTs) through the measurements of mass attenuation coefficients over a wide range of energies in comparison with graphite and the traditional shielding material lead.

\section{MATERIALS AND METHODS}

\subsection{Samples and radioactive sources}

Graphene and multi-walled nano tubeswere supplied by Anderlab Company were used. Single walled carbon nano tubes prepared at Prof. A.M. Rao's lab (Clemson University, USA)were used. Graphite powder was purchased from Sigma Aldrich. All the samples were compressed in order to form discs of $15 \mathrm{~mm}$ diameter; using an automatic pellet machine. graphene $(\mathrm{x}=0.1469$ $\left.\mathrm{gm} / \mathrm{cm}^{2}\right)$, graphite $\left(\mathrm{x}=0.2455 \mathrm{gm} / \mathrm{cm}^{2}\right)$, SWCNTs $(\mathrm{x}=0.2467)$, MWCNTs $\left(\mathrm{x}=0.2314 \mathrm{gm} / \mathrm{cm}^{2}\right)$. Approximately $10 \mathrm{mCi}$ radioactive sources ${ }^{60} \mathrm{Co},{ }^{133} \mathrm{Ba}$ and ${ }^{137} \mathrm{Cs}$ are obtained from Board of Radiation and Isotope Technology (BRIT), Bhabha Atomic Research Centre (BARC), Mumbai, India as liquid sources. Point sources have been made on Mylar backing. The sources have 
been covered with Mylar to avoid contamination of absorber materials during the study.

\subsection{Experimental set-up}

A good geometry set-up as shown in Fig.1 and an optimized and calibrated $\mathrm{NaI}(\mathrm{Tl})$ scintillation detector coupled to a PC based 8K Nuclear Multi Channel Analyzer (MCA) gamma ray spectrometer also shown in Fig.1 have been employed for recording the direct and absorbed photo peak spectra. The gamma spectra have been acquired using ${ }^{133} \mathrm{Ba},{ }^{137} \mathrm{Cs}$ and ${ }^{60} \mathrm{Co}$ radioactive sources for energies 356, 662, 1173 and $1332 \mathrm{keV}$ respectively. Typical data collection times were about 10,000 seconds for each measurement. The gamma spectra were analysed using GAMMA VISION software for the photo-peak areas [10].

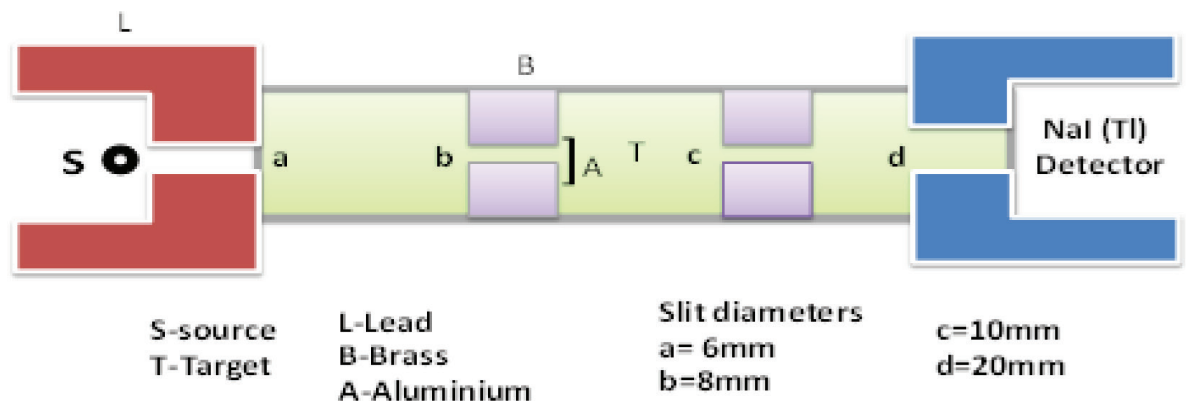

Figure 1: Experimental narrow beam good geometry set-up.

\subsection{Gamma transmission Method}

The attenuation of gamma radiation is due to the effect of all the energy exchange mechanisms such as Photoelectric effect, Pair production and Compton Effect. The transmitted intensity depends on the density, thickness of the absorbing layer and the cross-sectional properties of the material [11]. When gamma radiation of intensity $\mathrm{I}_{\mathrm{o}}$ is incident on a material of thickness $\mathrm{x}$, the attenuation of the gamma radiation by the material is given by the equation (1).

$$
I=I_{0} e^{-\mu x}
$$

Where $I_{0}$ is the number of counts recorded in the detector before attenuation, I is the number of counts recorded in the detector attenuation, $\mu$ is the linear attenuation coefficient, $\mathrm{x}$ is the thickness of the material measured in $\mathrm{cm}$. The values of $I$ and $I_{0}$ were averaged after recording 100 measurement points. A statistical fluctuation of gamma-ray beam intensities occurred within $1 \%$. We
Mass Attenuation Coefficient

Measurements of Some Nanocarbon

Allotropes: A New Hope for Better Low Cost Less-Cumbersome Radiation Shielding Over A Wide Energy Range 
Rajsekhar, E Narasimham, KL

Kurdekar, AD

Chunduri, LAA

Patnaik, S

Venkataramaniah, $\mathrm{K}$

used the well-known Beer-Lambert law to determine the mass attenuation coefficient of the carbon specimens,

$$
\frac{I}{I_{0}}=e^{\left(\frac{\mu}{\rho}\right) \rho x}
$$

Where $\rho$ is the material density, and $x$ the distance that the photon travels throughmatter (i.e., the thickness of the absorbing layer). Here we used the specimen density as the material density. The term $(\mu / \rho)$ is known as the mass attenuation coefficient $\mu_{m}$ and has been widely used as an intrinsic value shown in tables containing the gamma-ray mass attenuation coefficients [12].

\section{RESULTS AND DISCUSSION:}

The mass attenuation coefficients of MWCNTs, SWCNTS andgraphene are compared with the theoretical values for graphite and lead in Table 1. As can be seen, the mass attenuation coefficients of the nano carbons SWCNTs, MWCNTs and the Graphene are much larger that of bulk carbon by almost an order of magnitude. Also these nanocarbons seems to be surpassing the shielding property of the traditional shielding material, lead almost by a factor of two over all the energy values.

Table 1: Comparison of Mass Attenuation Coefficient (cm2/gm) of Nanomaterials.

\begin{tabular}{|c|c|c|c|c|c|}
\hline \multirow{2}{*}{ Material } & \multicolumn{4}{|c|}{ Mass Attenuation Coefficient (cm2/gm) } & \multirow{2}{*}{ Reference } \\
\hline & $356 \mathrm{keV}$ & $662 \mathrm{keV}$ & $1173 \mathrm{keV}$ & $1332 \mathrm{keV}$ & \\
\hline MWNT & $0.563 \pm 0.008$ & $0.383 \pm 0.005$ & $0.266 \pm 0.003$ & $0.251 \pm 0.003$ & Present work \\
\hline Graphene & $0.497 \pm 0.007$ & $0.364 \pm 0.005$ & $0.239 \pm 0.003$ & $0.216 \pm 0.003$ & Present work \\
\hline SWNT & $0.221 \pm 0.003$ & $0.135 \pm 0.002$ & $0.056 \pm 0.001$ & $0.042 \pm 0.001$ & Present work \\
\hline Lead & 0.283 & 0.141 & 0.061 & 0.052 & $\mathrm{XCOM}$ \\
\hline Graphite & 0.106 & 0.055 & 0.031 & 0.026 & $\mathrm{XCOM}$ \\
\hline
\end{tabular}

Fig. 2. shows the plotof mass attenuation coefficient of Nanomaterials at different energies. It is very interesting to note that for all the 4 energies studied, MWNTs and Graphene exhibited higher mass attenuation coefficients followed by lead, SWNT and graphite.

Based on the present results, MWNT and graphene can be considered as very good absorber material for gamma radiation shielding application when compared to other materials studied and even the traditional lead. It is also evident that as gamma energy increases gradually the mass attenuation coefficient of nano 


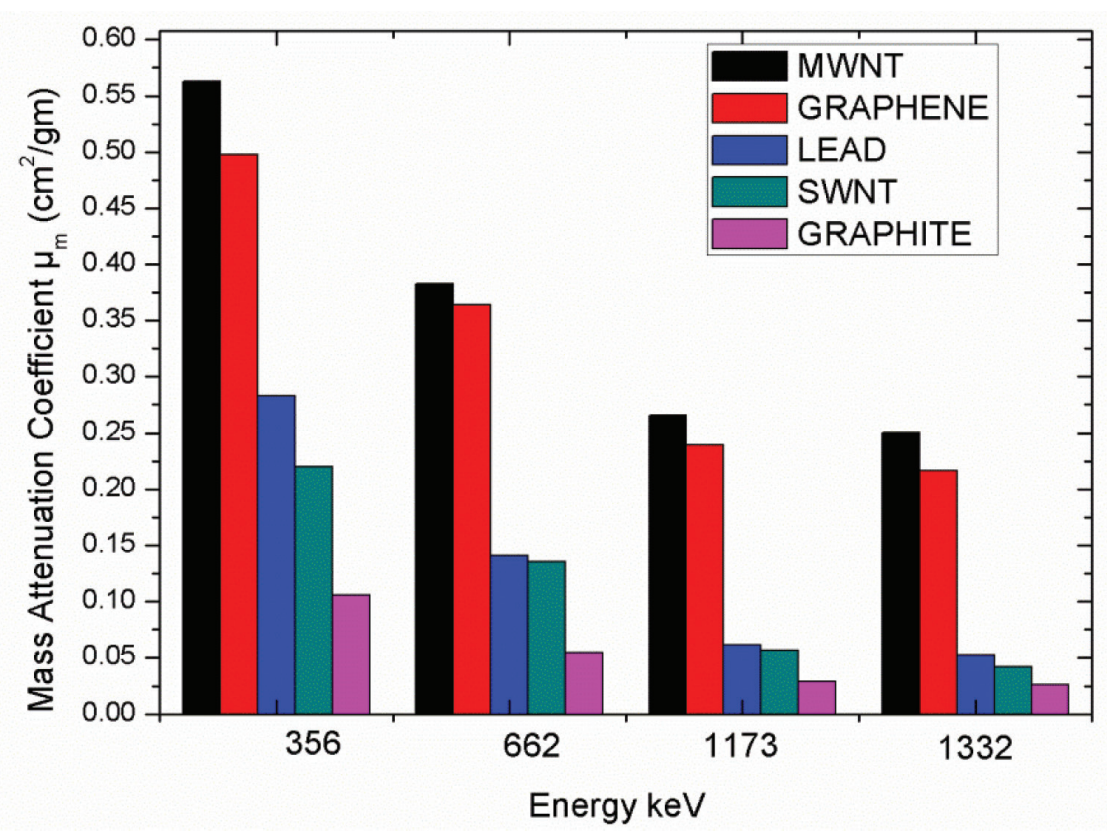

Figure 2: Plot of Mass attenuation coefficient of Nanomaterials at different energies.

materials also decreased. At all the energies MWNTs has the highest value of mass attenuation coefficient and graphite has the lowest value of mass attenuation coefficient compared to all other materials used. To validate our method we compared our measured mass attenuation coefficient of lead with XCOM data base and it is very close to the value reported in the XCOM data base.

Based on our observations MWNTs and graphene can be considered as very good absorber material for gamma radiation shielding can now open up new avenues for fabricating lightweight materials to offer protection against gamma-ray radiation.

As can be seen from the fig.2, among all the carbon allotropes, the nano carbons possessing nested cylindrical tubular structures exhibit a higher gammaray attenuation coefficient when compared to that of graphene and other carbon structures. It is interesting to note that the cylindrical carbon structures, nanotubes have increased the shielding capability significantly and it is more so with reference to MWCNTs. This phenomenon does not follow any conventional rule stating that the mass attenuation coefficients are elemental dependent. However, Fig. 2 also shows that the general trend of the decrease of mass attenuation coefficient as energy increases which is almost same for all the nanocarbons
Mass Attenuation

Coefficient

Measurements of

Some Nanocarbon

Allotropes: A

New Hope for

Better Low Cost

Less-Cumbersome

Radiation Shielding

Over A Wide

Energy Range

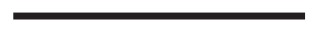


Rajsekhar, E Narasimham, KL

Kurdekar, AD

Chunduri, LAA

Patnaik, S

Venkataramaniah, $\mathrm{K}$ as well as bulk carbon and lead. In this account, we report that MWNT could efficiently absorb $\gamma$-rays when compared to graphene, lead, SWNT and graphite. Therefore, our results demonstrate that new models need to be revaluated, especially when describing the interactions between photons and electrons within the solid crystal lattice. It is noteworthy that the nanocarbons possessing cylindrical tube-like structures exhibit higher X-ray attenuation coefficient than that of bulk carbon. It can also be noted that the cylindrically rolled grapheme structures increase the gamma-ray attenuation significantly. This phenomenon does notfollow any conventional model, since the mass attenuation coefficients should bedetermined by the nuclei within the material rather than by their crystal structures [13].The mechanism for these observations is far from clear and it cannot be understood by conventional particle theory.

The unusually high shielding effect of nanocarbons is likely to originate from their unique cylindrical nano-morphology. A change in structure and morphology, at the nanoscale, could have a profound influence on macroscopic characteristics, through the paradigm of "function follows shape"[14]The nano structures could enhance the intrinsic electromagnetic properties, e.g., the dielectric constants and the electromagnetic interference EMI shielding. CNTs were shown to improve the EMI shielding of polymer composites to establish the predominant influence of intrinsic nanostructure geometry, e.g., aspect ratio and capacitive coupling, on electromagnetic characteristics.[15]. It was noted that while the projected length and diameter could influence the dielectric permittivity due to depolarization effects, the total extended length and diameter could determine EMI shielding. However, the length/diameter aspect ratio A.R.of the nanostructures does seem to make a difference. Generally, the depolarization electric field is enhanced [16] with a smaller A.R. and modifies the effective permittivity.

Garcia-Vidal et al. theoretically predicted that nanomaterial arrays could absorb light efficiently [17]. However, the hollow volumes and neighbouring materials are not considered in the model since their contribution in the derived equation is not significant. In general, the light absorption of nanomaterial is usually explained by the surface environment of the array rather than the morphology of the material itself [18]. These studies demonstrate that Nanomaterials could exhibit outstanding electromagnetic effects when compared to their bulk structures. An exhaustive study of this aspect with reference to materials such as $\mathrm{TiO}_{2}$ nanotubes and boron nitride nanotubes is the subject of our further investigations.

\section{ACKNOWLEDGMENT}

The authors wish to record their heartfelt gratitude to Bhagawan Sri Sathya Sai Baba for the beautiful facilities in the Nuclear Physics Research Laboratories of His University. 


\section{REFERENCES}

[1] T. Fujikawa and H. Arai, J. Elec. Spect. Relat. Phenom. 174 (2009) 85-92. https://doi.org/10.1016/j.elspec.2009.07.007

[2] T. Fujikawa, J. Elec. Spect. Relat. Phenom. 173 (2009) 51-78. https://doi.org/10.1016/j.elspec.2009.04.011

[3] K. Sawada, S.Murakami and N. Nagaosa, Phys. Rev. Lett. 96 (2006) 154802. https://doi.org/10.1103/PhysRevLett.96.154802

[4] A.N. Lagarkov, and A.K. Sarychev, Phys. Rev. B 53 (1996) 6318-6336. https://doi.org/10.1103/PhysRevB.53.6318

[5] Z. Peng, J. Peng and Y. Ou, Phys. Lett. A 359 (2006) 56-60. https://doi.org/10.1016/j.physleta.2006.05.076

[6] S.B. Tooski, J. Appl. Phys. 109 (2011) 14318-14324. https://doi.org/10.1063/1.3525059

[7] K.L. Dudley, R.W. Lawrence, Nano Lett. 5 (2005) 2131-2134. https://doi.org/10.1021/n1051375r

[8] Z. Liu, G. Bai, Yi. Huang, Y. Ma, F. Du, F. Li, T. Guo, Y.Chen, Carbon. 45 (2007) 821-827. https://doi.org/10.1016/j.carbon.2006.11.020

[9] A.L. Higginbotham, P.G. Moloney, M.C. Waid, J.G. Duque, C. Kittrell, H.K. Schmidt, J.J. Stephenson, S. Arepalli, L.L.Yowell, J.M. Your, Comp. Sci. and tech. 68 (2008) 3087-3092.

[10] Gamma Vision -32, 1998.Ver 5.10.EG \& G, ORTEC.

[11] I. Kaplan , Nuclear Physics, Addison-Wesley, New York, 1972.

[12] J.H. Hubbell, and S.M. Seltzer, NIST Standard Database 126, National Institute of Standards and Technology; Gaithersburg, MD, July 2004.

[13] J. Lu., D. Yuan, L. Jie, L. Weinan, E.K. Thomas, Nano Lett.. 8 (2008) 3325-3329. https://doi.org/10.1021/nl801744z

[14] P. R. Bandaru and A. M. Rao, JOM 59, 332007 Special Issue on Nanomaterials for Electronic Applications. https://doi.org/10.1007/s11837-007-0036-1

[15] S. H. Park, P. Theilmann, K. Yang, A. M. Rao, and P. R. Bandaru, Applied Physics Letters 96, 0431152010 https://doi.org/10.1063/1.3292214

[16] L. Landau, E. M. Lifshitz, and L. P. Pitaevskii, Electrodynamics of Continuous Media, 2nd ed. Butterworth-Heinemann, Boston, 1995.

[17] F.J. Garcia-Vidal, J.M. Pitarke, J.B. Pendry, Phys Rev Lett. 78 (1997) 4289-4292. https://doi.org/10.1103/PhysRevLett.78.4289

[18] Z. Ye, W.D. Deering, A. Krokhin, J.A. Roberts, Phys Rev B. 74 (2006) 075425-5. https://doi.org/10.1103/PhysRevB.74.075425
Mass Attenuation

Coefficient

Measurements of

Some Nanocarbon

Allotropes: A

New Hope for

Better Low Cost

Less-Cumbersome

Radiation Shielding

Over A Wide

Energy Range 